\title{
A SHORT OVERVIEW OF THE CYSTIC ECHINOCOCCOSIS AMONG PEOPLE IN BULGARIA AND STARA ZAGORA REGION DURING THE PERIOD 2006 - 2014
}

\author{
B. Chakarova ${ }^{1}$, K. Kichukova ${ }^{2}$, G. Rasheva ${ }^{3}$, Z. Naneva ${ }^{3}$, E. Kaneva ${ }^{3}$, T. Perchemlieva ${ }^{3}$, \\ S. Sabeva ${ }^{3}$ \\ ${ }^{1}$ Department of Hygiene, Epidemiology Infectious and Parasitic Diseases, Faculty of Medicine, \\ Trakia University, Stara Zagora, Bulgaria \\ ${ }^{2}$ Medical College, Trakia University, Stara Zagora, Bulgaria \\ ${ }^{3}$ Regional Health Inspectorate, Stara Zagora, Bulgaria
}

\begin{abstract}
The cystic echinococcosis among people in Bulgaria is a serious problem in human pathology. Echinococcosis is an uncommon disease in the EU, caused by infections with the larval stage of Echinococcus granulosus.

AIM: To analyze and compare the published data on the case rate of hydatid disease in Member States of the EC, some Balkan countries, in Bulgaria and in Stara Zagora region for a nine-year period (2006 - 2014) and to analyze and compare the published data on the case rate of hydatid disease in the member states of the EU and EEA, some Balkan countries and in Bulgaria. MATERIAL AND METHODS: Information from the cards of the epidemiological study of 140 patients operated from echinococcosis from Stara Zagora region has been used, as well as literature sources (annual reports on ECDC and scientific publications). Enclosed are an epidemiological study, biostatistical methods and analysis of results. RESULTS: In 2012, the case rate of echinococcosis was 0.19 cases per 100000 population in the EU and EEA. Bulgaria had the highest disease rate, 4.37 cases per 100000 people, and accounted for 39,2\% (320 confirmed cases) of the reported number of cases. During the period 2006 - 2012, the number of hydatid disease in Bulgaria are 2573 and constitute $42,63 \%$ of the population with hydatid disease in the EU and EEA $(n=6036)$. The case rate of echinococcosis in Bulgaria is 4.61 per 100000 population and in Stara Zagora region -4.67 per 100000 people. The case rate of echinococcosis by age group is as follows: children up to 14 years - 2.83 per 100000 population, from 15 to $64-4.1$ per 100000 people and over 65 years - 4.84 per 100000 population. The case rate for women is 4,21 per 100000 people and for men 4.19 per 100000 people. Incidence among citizens operated by echinococcosis people is 4.25 per 100000 people and 5.66 per 100000 people among the villagers. From those who have undergone an operation, $97.72 \%$ had a primary hydatid disease, and the organ localization is as follows: $77,7 \%$ in the liver; $20,4 \%$ in the lung and 1,9\% - in other locations. CONCLUSION: The invasions with cystic hydatid disease of the population in Bulgaria and in particular - the residents of Stara Zagora indicates serious deficiencies in the health awareness and behavior. This in conditions of prolonged socio-economic crisis leads to deterioration of health outcomes and quality of life of patients with hydatid disease .
\end{abstract}

Key words: Cystic echinococcosis, case rate of echinococcosis in Bulgaria, case rate of hydatid disease in Stara Zagora region

\section{INTRODUCTION}

The cystic echinococcosis among people in Bulgaria is a serious problem in human pathology. Its dynamic spreading supports trends unfavorable for public health and in

\footnotetext{
*Correspondence to: Borislava Georgieva Chakarova, Department of Hygiene, Epidemiology, Infectious \& Parasitic Diseases Faculty of Medicine, Trakia University, 6000 Stara Zagora, Bulgaria, Phone (++359 42) 664 233, Cell phone ++359888871 601, borislava_chakarova@abv.bg
}

economic terms $[1,2,3,4]$. Echinococcosis is an uncommon disease in the EU, caused by infections with the larval stage of Echinococcus granulosus. The annual reports of the ECDC (after 2006) analyze data reported by Member States of the EC on the surveillance of communicable diseases in the relevant country. After the inclusion of Bulgaria in the control system (2006) it was found out that our country permanently occupies one of the first places in case rate of hydatid disease in the European Union [5]. As 
a result of the successful implementation of the medical part of the National Programme for Control of Echinococcosis among humans and animals in Bulgaria (2004 - 2008) the decrease of morbidity almost doubled- from 8.32 per 100000 people (in 2002) to 4.37 per 100000 people (in 2012), $[6,7,8]$. The fact that from $1 / 4$ to $1 / 5$ of the cases are children and adolescents $(0-19$ years old $)$ is very worrisome and indicates contamination of the environment with parasite elements and active transmission of the parasitic zoonosis from the main sources - dogs to humans $[9,10]$. During the period 1990 - 2006 the highest case rate was registered in the following regions: Sliven (17.17 per 100000 people), Burgas (13.92 per 100000 people), Yambol (13.88 per 100000 people), Pazardzhik (11.97 per $100 \quad 000$ people) and Plovdiv (11.53 per $100 \quad 000$ people), [11]. During this period Stara Zagora region occupied one of the top five places in the country by case rate: 13.86 per 100000 people in 1994, 13.36 per 100000 people in 1995 and 10.08 per 100000 people in 1997 [12].

The aim of the study is to analyze and compare the published data on the case rate of hydatid disease in Member States of the EU, in some Balkan countries for a nine-year period (20062014).

\section{MATERIALS AND METHODS}

The information from the cards from the epidemiological study of 140 patients operated from echinococcosis from Stara Zagora region for nine-year period (2006 - 2014) has been used, as well as literature sources (annual reports on ECDC and scientific publications).

In order to calculate the indicators of morbidity, actual data of the National Statistical Institute for the demographic structure of the population of Stara Zagora region was used. Enclosed are an epidemiological study, biostatistics methods and analysis of the results.

\section{RESULTS AND DISCUSSION}

Cystic echinococcosis accounts for $95 \%$ of the estimated 2-3 million cases of human echinococcal infections worldwide and represents a major public health problem in many parts of the world [13]. It is listed by the World Health Organization as a neglected zoonotic disease since this disease mainly affects poor and marginalized populations in low-resource settings [14].WHO is working towards the validation of effective cystic echinococcosis control strategies by 2020 [15]. Italy, Spain and Eastern European countries (such as Bulgaria, Romania and Turkey) are recognized as highly endemic $[16,17]$. In USA for the years 1996-2003 Disability-adjusted life years (DALYs) and monetary losses, resulting from human and livestock $\mathrm{CE}$, are calculated from recorded human and livestock cases. Alternative values, assuming substantial underreporting, are also reported. When no underreporting is assumed, the estimated human burden of disease is an annual loss of US $\$ 193,529,740$. When underreporting is accounted for, this amount rises to $1,009,662$ DALYs or US $\$ 763,980,979$ [4].

During the period 2006 - 2012, the number of cases of cystic hydatid disease in the EU and EEA was 6036 and in Bulgaria was 2573, representing $42,63 \%$ of all European cases (Figure 1). Especially disturbing is the fact that in 2006 the cases of hydatid disease in Bulgaria represented approximately $50 \%$ of those in the EU (Table 1),[5]. The situation on the Balkan peninsula concerning hydatid disease varies in different countries (Figure 2). In the early 20th century in Croatia cases per 100000 people was 28.3 and at the end of the century it dropped down to 4.2. In 1984 Greece launched a National program for fight againt echinococcosis and from 12.0 per 100000 people in 80 s of XX century it was reduced to 3.5 per 100000 people [18-21].

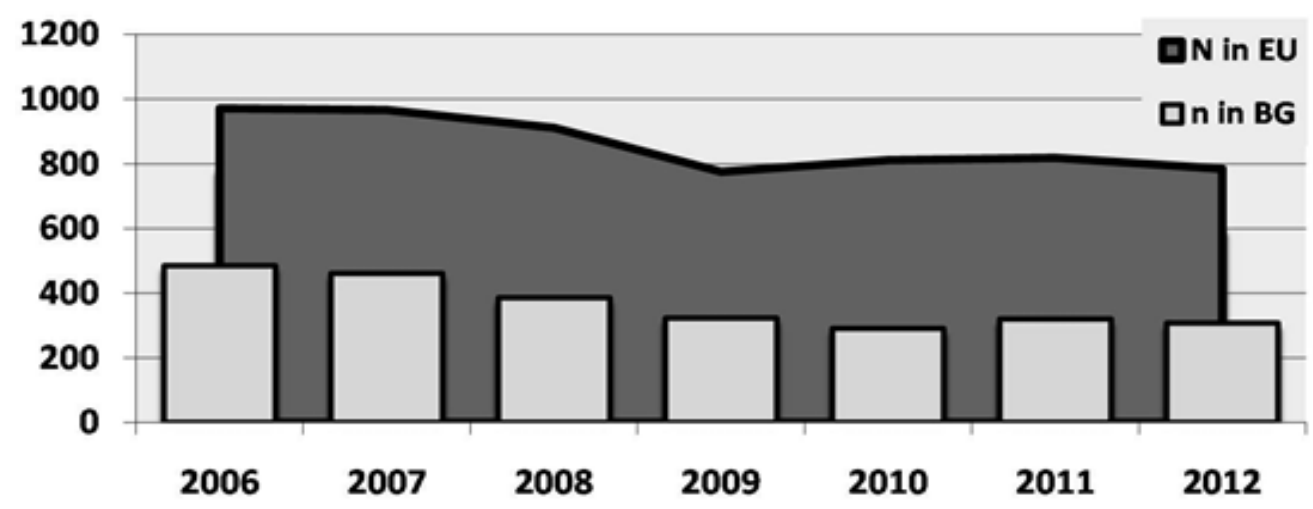

Figure 1. Number of reported confirmed echinococcosis cases in EU countries and in Bulgaria during the period 2006-2012 [ECDC, 2014] 
CHAKAROVA B., et al.

Table 1. Number, rate and \% of reported confirmed echinococcosis cases in EU countries and in Bulgaria, 2006-2012

\begin{tabular}{|cccccc|}
\hline Year & N inEU & $\begin{array}{c}\text { cases per } \\
\text { (100000 }\end{array}$ & n in BG & $\begin{array}{c}\text { cases per } \\
\text { (00000 }\end{array}$ & $\begin{array}{c}\text { cases } \\
\text { in BG/EU [\%] }\end{array}$ \\
\hline $\mathbf{2 0 0 6}$ & 971 & 0,23 & 485 & 6,28 & 49,95 \\
$\mathbf{2 0 0 7}$ & 966 & 0,22 & 461 & 6 & 47,72 \\
$\mathbf{2 0 0 8}$ & 911 & 0,21 & 386 & 5,13 & 42,37 \\
$\mathbf{2 0 0 9}$ & 775 & 0,18 & 323 & 4,33 & 41,68 \\
$\mathbf{2 0 1 0}$ & 811 & 0,17 & 291 & 3,92 & 35,88 \\
$\mathbf{2 0 1 1}$ & 818 & 0,18 & 320 & 4,17 & 39,12 \\
$\mathbf{2 0 1 2}$ & 784 & 0,19 & 307 & 4,37 & 39,16 \\
\hline Total & $\mathbf{6 0 3 6}$ & & $\mathbf{2 5 7 3}$ & & $\mathbf{4 2 , 6 3 \%}$ \\
\hline
\end{tabular}

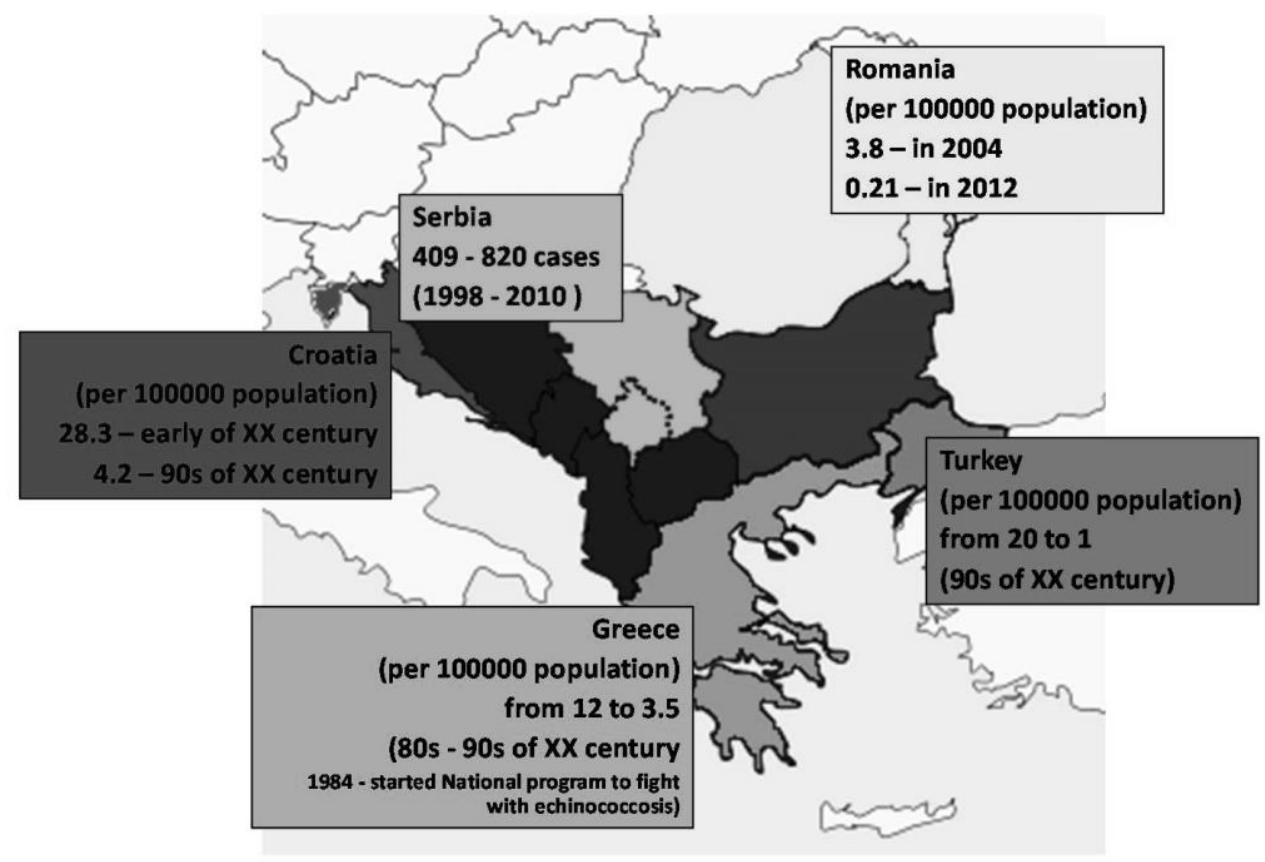

Figure 2. Echinococcosis among the population of some countries on the Balkan Peninsula

Stara Zagora region is located in Central South Bulgaria and is among the six largest districts in the country. Its territory is $5151.1 \mathrm{~km} 2$ with a population of 333265 inhabitants [22-24]. The population density is relatively high, about 65 people per $\mathrm{km} 2$. In the area approximately $5 \%$ of the total population of the country is concentrated and under this indicator the region ranks fifth. Two thirds of the population lives in the cities of region. The largest ones are: Stara Zagora, Kazanlak, Chirpan, Radnevo. Stara Zagora comprises 206 settlements: 10 towns and 196 villages. There are 11 municipalities functioning in Stara Zagora region, which are very different in terms of provision, resources, economic and infrastructural potential [23].

The number of registered and operated patients with hydatid disease in Stara Zagora region over a nine-year period (2006 - 2014) is 140 . The highest case rate was registered in 2008 6.6 per 100000 people and in $2010-5.7$ per 100000 people and the lowest one being in 
2014 - 1.8 per 100000 people. For 2013 and 2014 there is no official published data regarding cases of hydatid disease in Bulgaria (Figure 3). As a result of the successful implementation of the medical part of the National Programme for Control of echinococcosis in humans and animals in Bulgaria (2004 - 2008) there was decrease in the number of registered cases of cystic hydatid disease [6]. The data for significant decrease of the cases in 2014 in Stara Zagora region is probably inaccurate. This may be due to problems with the communication between the Regional Health Inspectorate (RHI) - Stara Zagora and the medical institutios where the patients have been operated. The reasons for this are multiple, including but not limited to the structural transformations of hospitals and reorganization of the RHI (consolidation of departments and divisions, relocation of whole medical teams from one hospital to another, etc.).

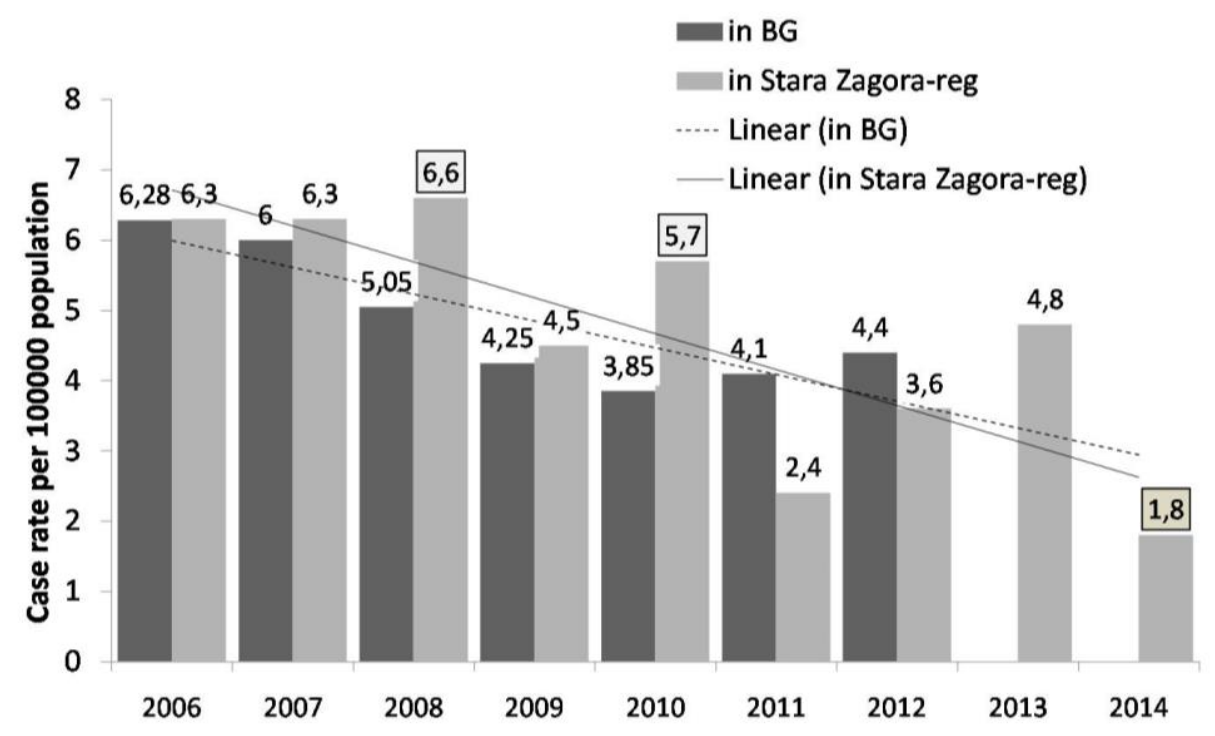

Figure 3. Dynamics and trend of case rate of hydatid disease among the population in Bulgaria and Stara Zagora region during the period 2006 - 2014

For the period 2006 - 2014 the average annual incidence in Stara Zagora region was 4.67 per 100000 people. From the eleven municipalities of Stara Zagora region in two of them (Maglizh and Galabovo) case rate is significantly above the regional average: 14.0 per 100000 people and 8.5 per 100000 people respectively. In the municipality of Bratya Daskalovi (3.3 per 100000 people) and Chirpan (2.6 per 100000 people) the case rate is below average. In the other seven municipalities the case rate is around and above average (Figure 4). Probably the number of cases of hydatid disease is considerably higher. In the municipalities with the biggest number of operated people there are compact Roma communities with high unemployment rates and low social status. The aggregate of human risk factors and the unsolved problem with the large populations of street dogs wandering without anthelmintic treatment makes echinococcosis socially significant disease.

The distribution of the operated patients with hydatid disease in the Stara Zagora region by age groups is presented in Figure 5. The biggest is the proportion of patients aged over
65 years $-29,6 \%$, followed by the group of children and adolescents (from 0 to 19 years) $21,4 \%$. The relative share of patients in the age group 0 to 14 is $12,2 \%$, with case rate of 3.8 per 100000 people. Patients in the active age constitute $63,3 \%$ of the operated ones, with morbidity rate of 4.1 per 100000 people, and those over the age of $65-22,4 \%$, morbidity rate of 4.8 per 100000 people. These indicators correspond to the statistics for the aging of the Bulgarian nation and to the age pathology respectively. The high levels of case rate of echinococcosis in the age over 65 are often associated with the late diagnosis at the stage of complications. The fact that approximately $1 / 5$ of the cases are children and adolescents ( 0 - 19 years) is very worrisome and indicates presence of contamination of theenvironment with parasitic elements and very active transmission of the parasitic zoonosis from the main sources - dogs, to humans $[25,26]$. As for the entire country, as well as the region of Stara Zagora, the large proportion of echinococcosis in children and adolescents is a signal for the presence of active parasitic outbreaks with continuing intense transmission of the invasion, which is a bad prognostication sign. The cases of hydatid 
disease are usually sporadic, but often is "family echinococcosis" especially often discovered in migrant communities with low
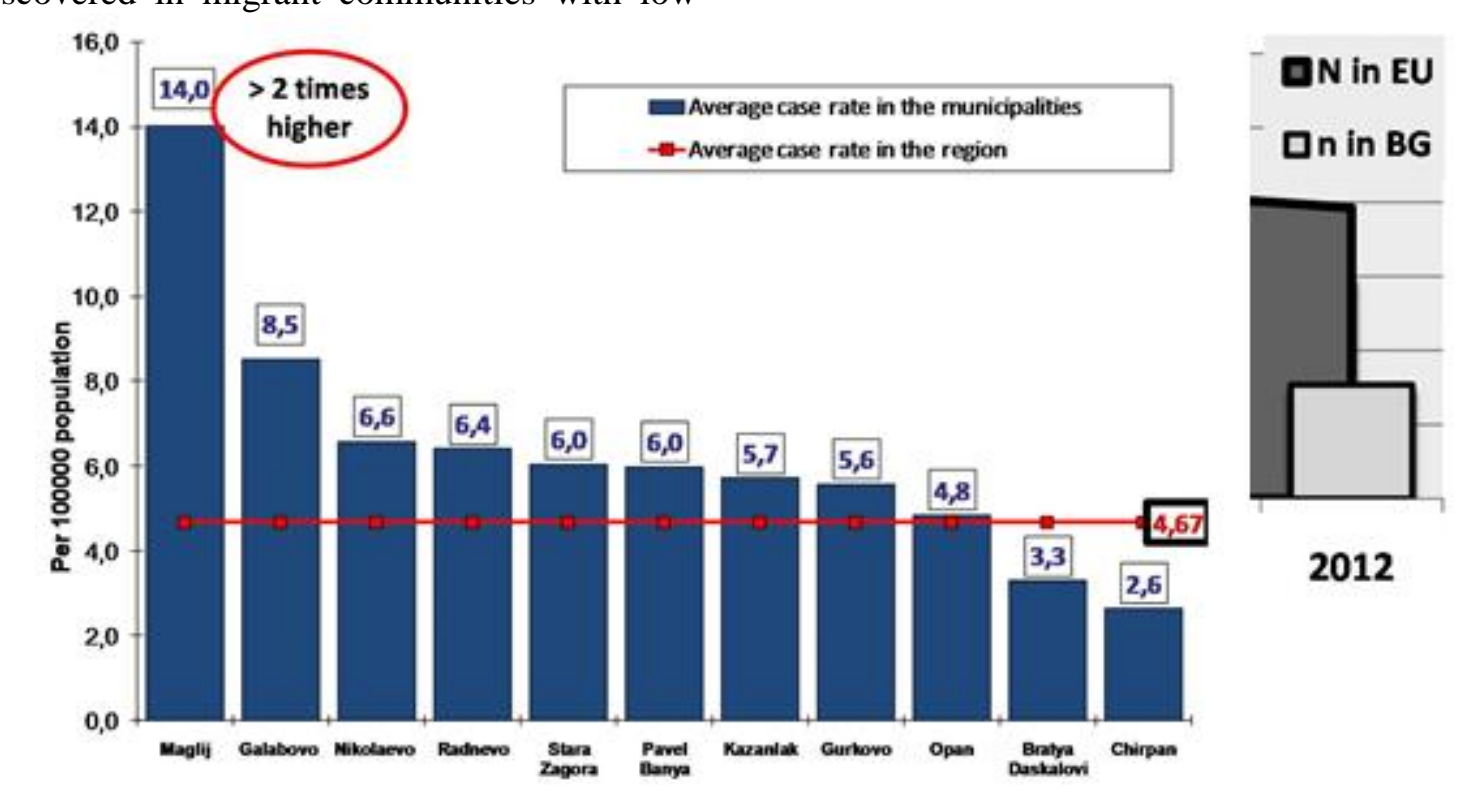
sanitary minimum.

Figure 4. Case rate of hydatid disease among people in municipalities Stara Zagora region during the period $2006-2014$

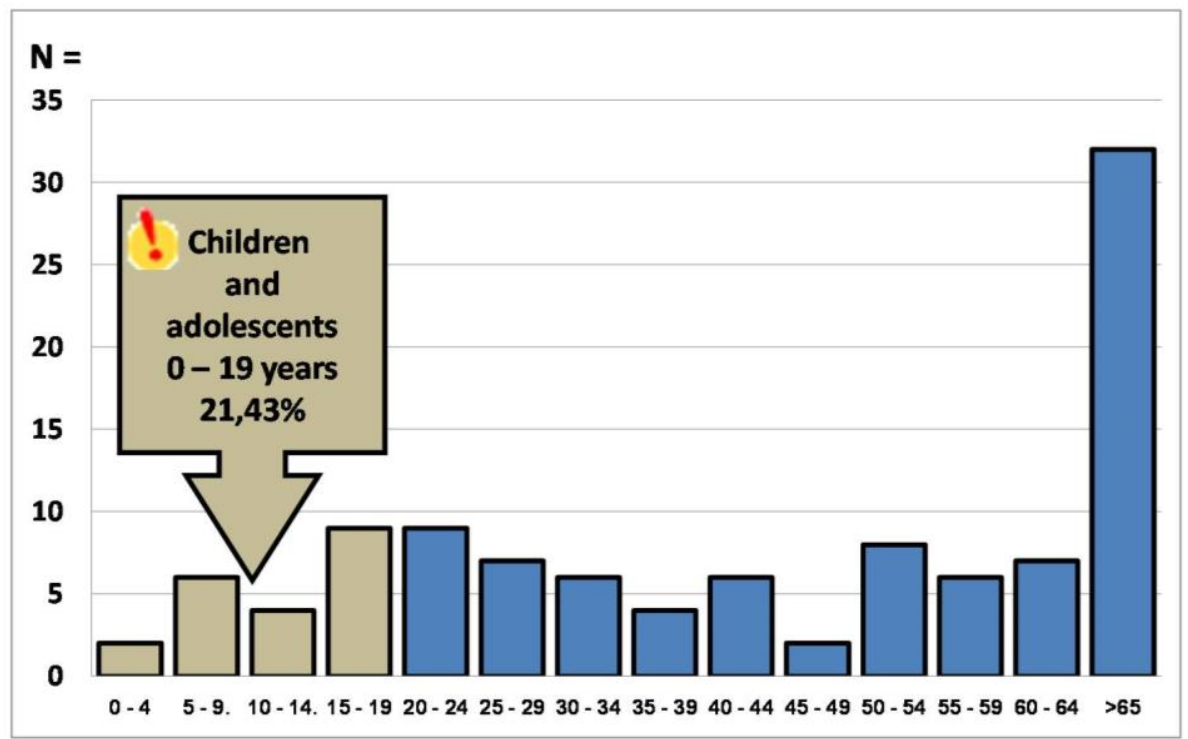

Figure 5. Structure of echinococcosis by age groups in Stara Zagora region during the period 2006 - 2014

The distribution by gender shows that the case rate is evenly represented among both genders - 4.18 per 100000 people among female patients and 4.21 per 100000 people among the male ones.

The distribution of the operated patients with of echinococcosis by place of residence shows a large proportion of people living in the city center of the municipality $-64,3 \%$, compared with the proportion of patients living in rural areas - $35,7 \%$, but the incidence in rural residents from 5.66 per 100000 people is statistically higher than that of urban residents - 4.25 per 100000 people. This difference in the levels of case rate is clearly supported by the accumulation of risk factors in the rural areas - lack of knowledge of breeding of domestic animals, absence of anthelmintic prevention and last but not least - rural depopulation and the related decline of the standard of living.

According to data from cards for the epidemiological study of patients operated by of echinococcosis, the organ localization of hydatid cysts is most often in the liver - in $74,4 \%$ of cases, in the lungs $-23,1 \%$ and in the spleen $-2,6 \%$. These data, however, diverge from clinical observations in which there are cysts also in the bones - in 2 patients, in the brain - in one child. Perhaps patients were 
operated in hospitals which did not report about the surgical treatment of echinococcosis, which is why cases are not registered. In the majority of cases $(96,3 \%)$ echinococcosis is represented by a single larval form and only in $3,3 \%$ of cases - of multiple hydatid. The removed cysts mostly have sizes between 6 and $8 \mathrm{~cm}$ (in 21 cases) and between 1 and $5 \mathrm{~cm}$ (in 21 cases).

Literature shows similar proportionality in organ location of echinococcosis cyst. The outcome of the disease depends on the location of the cysts, their relationship with neighboring organs, size and stage of maturity and possible complications [27].

\section{CONCLUSIONS}

1. Echinococcosis in Stara Zagora region is a socially relevant zoonozis.

2. According to the case rate during the period 2006 - 2014 the region occupies an intermediate position but is higher than the average for Bulgaria.

3. The relative share of operated children and teenagers is about $1 / 5$ of all suffering from echinococcosis, which is an indicator of high contamination of the environment with eggs of E. granulosus, or for low culture of health and risky behavior respectively.

4. The incidence of hydatid disease among men and women is approximately equal, which is associated with the high rate of long-term unemployment in the regions with the highest growth of the zoonosis.

5. The relative share of urban residents with hydatid disease is twice as high as that of rural residents, but incidence in rural areas is significantly higher.

6. Echinococcosis continues to be one of the most serious parasitic diseases in Bulgaria. The deteriorated socioeconomic status and the resultant permanent poverty of most of the population requires the inclusion of risk contingents in preventive programs and active detection and treatment of the diseased and sanitation of the environment.

\section{REFERENCES}

1. Nikiforov, St., Boeva V., Echinococcosis. Sofia, Medicine and Sport, 1983

2. Todorov T., V. Boeva., Human echinococcosis in Bulgaria: a comparative epidemiological analysis. Bulletin of the World Health Organization, 77, 110-118, 1999.

3. Moro P., Schantz P.M., Echinococcosis: a review. International Journal of Infectious Diseases 13, 125-133, 2009.

4. Budke C.M., P.Deplazes, P.R.Torgerson. Global Socioeconomic Impact of Cystic
CHAKAROVA B., et al.

Echinococcosis. Emerging Infectious Diseases, 12, 2, 296-303, 2006.

5. European Centre for Disease Prevention and Control. Annual epidemiological report 2014 - food- and waterborne diseases and zoonoses. Stockholm: ECDC; 32-33, 2014.

6. National Programme for Control of echinococcosis in humans and animals (2004-2008), Veterinary collectible, 2005

7. Raynova I., D.Yordanova, I.Harizanov, I.Marinova, I.Bikov, I.Kaftandjiev N.Tsvetkova. Parasitic diseases in Bulgaria in 2011. Distribution and analysis. Information Journal of the NCIPD, 2, 410,2012.

8. Rainova I., I.Marinova, R.Harizanov, D.Jordanova,I.Kaftandjiev, I.Bikov, N.Tsvetkova. Parasitic diseases in Bulgaria in 2012. Problems of Infectious and Parasitic Diseases, NCIPD, 42,1,28-40, 2014.

9. Todorov T., V.Boeva. Echinococcosis in children and adolescents in Bulgaria: a comparative study. Anals of Tropical Medicine and parasitology, 94, 2, 135-144, 2000.

10.Djuricic S.M., Grebeldinger S., Kafka D.I., Djan I., Vukadin M., Vasiljevic Z.V., Cystic echinococcosis in children - Then seventeen-year experience of two large medical centers in Serbia. Parasitology International 59, 257-261,2010.

11. Marinova, I., D. Yordanova, R. Kurdova. Echinococcosis In: Parasitic zoonoses in humans. Edited by R. Kurdova. NCIPD, Sofia, 8-49, 2008.

12.Petrov P., M.Halacheva. Epidemiological organizational problems echinococcosis in humans and farm animals. Information Journal of the NCIPD, 7, 22-28, 1996.

13.Craig P.S., Budke C.M., Schantz P.M., Li T., Qiu J., Yang Y., Zeyhle E., Rogan M.T., Ito A., Human Echinococcosis: A Neglected Disease?, Tropical Medicine and Health, 35, 4, 283-292, 2007.

14.www.who.int/neglected_diseases/zoonoses

15.www.who.int/mediacentre/factsheets/fs377/ en/

16.http://www.who.int/mediacentre/factsheets/ fs $377 / \mathrm{en} /$

17.http://www.heraclesfp7.eu/background.html

18.Ozcel A.M., Department of Parasitology, Faculty of Medicine, University of Ege, Izmir, Turkey. Country report. MZCP Consultation on the E/H National Control Programmes in the MZCC countries, Valladolid, Spain, 1994.

19.Altintas N., Past to present: echinococcosis in Turkey. Acta Trop., 85,2:105-12, 2003. 
20.Echinococcosis in Europe - FAO.org., Vuitton Dominique A., Economides P., and the WHO-IWGE EurEchinoReg Network. Echinococcosis in Western Europe, a risk assessment/risk management approach. Charissis, Ministry of Agriculture of Greece, c/o MZPC Greece, personal communication, 1999.

21.Calma C.L., Neghina A.M., Vlaicu B., Neghina R., Cystic echinococcosis in the human population of a western Romanian county, 2004-2010. Clinical Microbiology and Infection, 17, 11, 1731-1734, 2011.

22.http://censusresults.nsi.bg/Census/Reports/ Oblast.aspx?Obl=SZR

23.http://www.sz.government.bg/?go=t\&id=11 $\& \operatorname{mbtn}=1120 \& \operatorname{mgid}=1 \&$ selm $=1150$
24. National Statistics Institute, 2012. Guide to Stara Zagora region. Census of population and housing in 100, 2011.

25. Boeva-Bangyosova, V.B., Z.E. Ivanova. Current status and prospects of echinococcosis control in the Republic of Bulgaria. Bulgarian Journal of Veterinary Medicine, 2, 4, 177-190, 1999.

26. Boeva-Bangyozova V. and V. Bangyozov. Health care reform and parasitosis among people in Bulgaria. Social Medicine, 4, 8$12,2012$.

27.Popova-Daskalova, P., D. Vutchev, D. Stantcheva. Echinococcosis in Central South Bulgaria - Plovdiv and Pazardzhik Region (2009-2013), Social Medicine, 2022, 4, 2013 